\title{
Inhaled corticosteroids in COPD: the final verdict is...
}

\author{
Janice M. Leung and Don D. Sin \\ Affiliation: Centre for Heart Lung Innovation, St Paul's Hospital and Division of Respiratory Medicine, \\ University of British Columbia, Vancouver, BC, Canada.
}

Correspondence: Don D. Sin, Room 348, Burrard Building, St Paul's Hospital, Vancouver, BC, V6Z 1Y6, Canada. E-mail: don.sinahli.ubc.ca

@ERSpublications

History (and features) of asthma, along with peripheral blood eosinophil counts, can guide provision of inhaled corticosteroids in COPD management http://ow.ly/uApH30mgEh4

Cite this article as: Leung JM, Sin DD. Inhaled corticosteroids in COPD: the final verdict is.... Eur Respir J 2018; 52: 1801940 [https://doi.org/10.1183/13993003.01940-2018].

Inhaled corticosteroids (ICS) were first discovered 50 years ago [1] and are now the most widely used controller medication in the world for chronic obstructive pulmonary disease (COPD). Over the past two decades, $>40000$ patients with COPD have participated in large-scale ICS therapeutic trials. What have we learned from these studies?

First, ICS reduce the risk of exacerbations but the overall benefits are relatively modest. Second, ICS therapy can induce harm. The most notable and unique to COPD is the increased risk of pneumonia especially among those with moderate to severe disease. Third, ICS combined with a long-acting $\beta_{2}$-agonist (LABA) is more effective in improving symptoms and reducing exacerbations than each drug alone; however, it is debatable whether the ICS/LABA combination is superior to long-acting muscarinic antagonist (LAMA) alone or in combination with a LABA (i.e. LABA/LAMA) [2]. In clinical practice, the most common COPD patients requiring pharmacotherapy are symptomatic patients (defined by a COPD Assessment Test score $\geqslant 10$ ) with two or more exacerbations in the previous year [3]. For these patients, the Global Initiative for Chronic Obstructive Lung Disease (GOLD) committee recommendations are ambiguous, offering clinicians a choice of LAMA alone, LABA/LAMA, ICS/LABA or ICS/LABA/LAMA combinations [2]. Thus, currently, therapeutic decisions are driven largely by personal preference (or habit) rather than "evidence". Given the long history of ICS and the widespread availability of these drugs, it is not surprising that ICS remains the top choice for many clinicians in the community for the management of COPD patients. This "historic medicine" approach to ICS, however, has been heavily criticised by experts for being wasteful, inefficient and imprecise [4]. Is there a way to usher in the era of "precision medicine" for our patients with COPD?

Four papers in this issue of the European Respiratory Journal (ERJ) provide some important information that may enable targeting of ICS to the "right" patients at the "right" time and get us closer to the holy grail of "precision medicine" in COPD. First, ICS should be provided for COPD patients who also have asthma (i.e. asthma-COPD overlap) [5]. As pointed out by SuISSA and ARIEL [6] in their very provocative and interesting commentary, the decision to include (or exclude) asthma-COPD overlap patients may explain why some studies of ICS-containing "double" or "triple" therapies were "positive" while others were "negative". The IMPACT [7] and TRIBUTE [8] trials, for instance, enrolled patients with a prior but not current history of asthma, and both found a reduced rate of moderate to severe exacerbations among those treated with ICS-containing combinations. In contrast, in the FLAME trial [9], COPD patients with 
a history of asthma or features of asthma were excluded and the study showed that LABA/LAMA was associated with lower rates of exacerbations than the ICS/LABA combination.

The analysis by SUISSA and ARIEL [6] revealed another interesting observation in these trials. In the first month following ICS withdrawal, there was a transient surge in the exacerbation rate in the ICS-free arms (compared with the rate observed in the ICS-containing arms) with the rate returning back to "normal" levels thereafter, resulting in no difference in exacerbation rates between the ICS-containing and ICS-free arms by the end of the study period (figure $1 \mathrm{a}$ and $\mathrm{b}$ in [6]). This first month exacerbation surge was particularly notable in the IMPACT trial, which did not supplement patients with either LAMA or LABA/ LAMA during the first few weeks of ICS withdrawal, which may have resulted in exacerbation or exacerbation-like events related to sudden discontinuation of ICS.

Second, as noted previously, ICS can increase the risk of pneumonia. There has been considerable debate on the relevance of these pneumonia events as they do not appear to materially affect mortality. This conundrum (i.e. balancing the benefits of ICS for exacerbation against the potential harm of pneumonia) is addressed by CAZZOLA et al. [10] who used the SUCRA (surface under the cumulative ranking curve) method to systematically rank order ICS and non-ICS based therapies, taking into consideration their benefits as well as their risks. They found that the overall SUCRA values favoured LABA/LAMA combination over ICS/LABA/LAMA and LABA or LAMA alone. However, as noted by AGUSTI et al. [11], the effects of ICS therapy are modified by peripheral blood eosinophil count. Patients with blood eosinophil counts of $\geqslant 300$ cells per $\mu \mathrm{L}$ are much more likely to be responsive to ICS-based therapy than those with lower eosinophil counts. This important observation was replicated in the analysis by CAzzolA et al. [10]. In that analysis, the number needed to treat (NNT) for ICS/LABA/LAMA compared to LABA/ LAMA treatment was only 8-10 patients to prevent a patient from experiencing a significant exacerbation over 1 year. For those with blood eosinophil counts $<300$ cells per $\mu \mathrm{L}$, however, the NNT was $\sim 47$, which is likely unacceptable for most clinicians.

Third, as reported by AGUSTI et al. [11], there are significant risk factors associated with ICS-related pneumonia. These include advanced age, reduced body mass index, severe airflow limitation (GOLD grades 3 and 4), low blood eosinophil count or a prior history of mycobacterial disease. As such, ICS therapy should be used very judiciously in these patients (if at all) and if they develop recurrent bouts of pneumonia, ICS should probably be discontinued or switched to lower potency formulations, which as noted by CAzzola et al. [10], are associated with much lower risk of pneumonia.

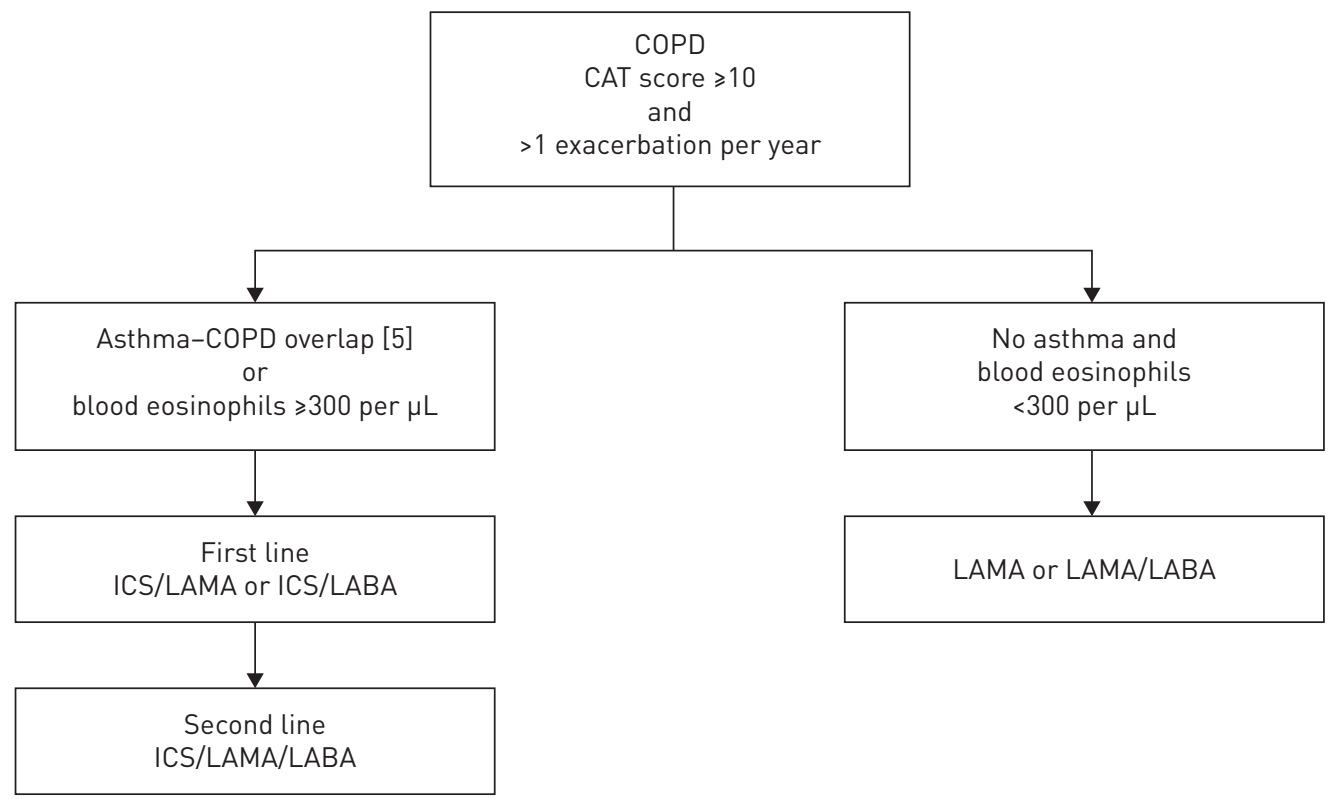

FIGURE 1 A proposed approach to management of symptomatic chronic obstructive pulmonary disease (COPD) patients with a significant history of exacerbations. Patients should first be evaluated to determine whether they have asthmatic features (e.g. history of asthma, large bronchodilator response and others; see [5] for other characteristics) and/or demonstrate blood eosinophil count $\geqslant 300$ cells per $\mu \mathrm{L}$. If they fulfil any of these criteria, the first-line therapy includes an inhaled corticosteroid (ICS)-containing compound. If patients do not fulfil these criteria, then the first line therapy is a long-acting muscarinic antagonist (LAMA) alone or a long-acting $\beta_{2}$-agonist (LABA)/LAMA based on availability, cost and prior responses. CAT: COPD Assessment Test. 
Based on our interpretation of the current evidence at hand, we have summarised an approach for managing symptomatic patients with a significant history of exacerbations in figure 1 . We believe that patients should first be assessed for asthmatic features including a prior history of asthma, bronchodilator reversibility (or bronchoprovocation challenge test where appropriate) and atopic status. For those with asthma-COPD overlap [5], we recommend ICS-based therapy in a stepwise fashion, starting with ICS/ LABA or ICS/LAMA, which can be escalated to ICS/LABA/LAMA should patients continue to experience exacerbations or remain highly symptomatic. For those without asthmatic features, their blood eosinophil count should be measured, perhaps more than once. For those with eosinophil count $\geqslant 300$ cells per $\mu \mathrm{L}$, they may be treated in a similar manner to those with asthma-COPD overlap. For those with blood eosinophils $<300$ cells per $\mu \mathrm{L}$, the first line is either LAMA alone or a LABA/LAMA combination, depending on cost, availability, patient preference and prior responses.

Notwithstanding the importance of the four studies in this issue of ERJ, there are many unresolved questions regarding the role of ICS in COPD management. Although both SUMMIT [12, 13] and TORCH [13] failed to demonstrate a survival benefit of ICS-based therapy, the pooled analysis by Vestвo et al. [14] raises the possibility that the newer generation of ICS-based therapies based on ultrafine delivery devices may prolong survival of COPD patients, especially those with comorbidities. Future studies will need to rigorously test this hypothesis. Beyond mortality, the effects of ICS-based therapy on hospitalisations, which are the largest drivers of direct costs for COPD care in the western world, remain largely unknown. Finally, although blood eosinophils are a good biomarker to guide ICS therapy, they do not appear to play an important role in the pathogenesis of COPD and as such are not good therapeutic targets [15]. There is a pressing need to better understand the pathogenesis of COPD and identify novel therapeutic targets (with accompanying robust response biomarkers) to modify the "natural history" of COPD and bring precision medicine to the bedside of millions of patients with COPD around the world.

Conflict of interest: J.M. Leung has nothing to disclose. D.D. Sin reports receiving a grant from Merck to provide funding for an investigator-initiated project to ascertain genomic determinants of COPD; honoraria for advisory meetings on COPD from Sanofi-Aventis and Regeneron; grants from Boehringer Ingelheim to provide research funding for clinical trials and an investigator-initiated study on the effects of olodaterol on airway inflammation; a grant from AstraZeneca to provide funding for clinical trials, and honoraria for speaking engagements and sitting on COPD advisory boards; and honoraria for speaking engagements and sitting on advisory boards from Novartis.

Support statement: J.M. Leung is supported by the Michael Smith Foundation for Health Research, the Providence Health Care Research Institute and the CIHR-AstraZeneca Early Career Investigator Award. D.D. Sin is a Tier 1 Canada Research Chair in COPD and holds the De Lazzari Family Chair at the Centre for Heart Lung Innovation (St Paul's Hospital and Division of Respiratory Medicine, University of British Columbia, Vancouver, BC, Canada).

\section{References}

1 Brown HM, Storey G, George WH. Beclomethasone dipropionate: a new steroid aerosol for the treatment of allergic asthma. $\mathrm{Br}$ Med J 1972; 1: 585-590.

2 Vogelmeier CF, Criner GJ, Martinez FJ, et al. Global Strategy for the Diagnosis, Management, and Prevention of Chronic Obstructive Lung Disease 2017 Report: GOLD Executive Summary. Eur Respir J 2017; 49: 1700214.

3 Han MK, Quibrera PM, Carretta EE, et al. Frequency of exacerbations in patients with chronic obstructive pulmonary disease: an analysis of the SPIROMICS cohort. Lancet Respir Med 2017; 5: 619-626.

Schork NJ. Personalized medicine: time for one-person trials. Nature 2015; 520: 609-611.

5 Sin DD, Miravitlles M, Mannino DM, et al. What is asthma-COPD overlap syndrome? Towards a consensus definition from a round table discussion. Eur Respir J 2016; 48: 664-673.

6 Suissa S, Ariel A. Triple therapy trials in COPD: a precision medicine opportunity. Eur Respir J 2018; 52: 1801848

7 Lipson DA, Barnhart F, Brealey N, et al. Once-daily single-inhaler triple versus dual therapy in patients with COPD. N Engl J Med 2018; 378: 1671-1680.

8 Papi A, Vestbo J, Fabbri L, et al. Extrafine inhaled triple therapy versus dual bronchodilator therapy in chronic obstructive pulmonary disease (TRIBUTE): a double-blind, parallel group, randomised controlled trial. Lancet 2018; 391: 1076-1084

9 Wedzicha JA, Banerji D, Chapman KR, et al. Indacaterol-glycopyrronium versus salmeterol-fluticasone for COPD. N Engl J Med 2016; 374: 2222-2234.

10 Cazzola M, Rogliani P, Calzetta L, et al. Triple therapy versus single and dual long-acting bronchodilator therapy in COPD: a systematic review and meta-analysis. Eur Respir J 2018; 52: 1801586.

11 Agusti A, Fabbri LM, Singh D, et al. Inhaled corticosteroids in COPD: friend or foe? Eur Respir J 2018; 52: 1801219.

12 Vestbo J, Anderson JA, Brook RD, et al. Fluticasone furoate and vilanterol and survival in chronic obstructive pulmonary disease with heightened cardiovascular risk (SUMMIT): a double-blind randomised controlled trial. Lancet 2016; 387: 1817-1826.

13 Calverley PM, Anderson JA, Celli B, et al. Salmeterol and fluticasone propionate and survival in chronic obstructive pulmonary disease. N Engl J Med 2007; 356: 775-789.

14 Vestbo J, Fabbri L, Papi A, et al. Inhaled corticosteroid containing combinations and mortality in COPD. Eur Respir J 2018; 52: 1801230.

15 Pavord ID, Chanez P, Criner GJ, et al. Mepolizumab for eosinophilic chronic obstructive pulmonary disease. N Engl J Med 2017; 377: 1613-1629. 